Chapter 4

\title{
Cooperative Effects of Neuronal Nitric Oxide Synthase and Endothelial Nitric Oxide Synthase on Gastric Hyperemic Response to Intragastric Capsaicin
}

\author{
Syunji Horie, Masaki Raimura, Kenjiro Matsumoto, \\ Takao Namiki, Katsutoshi Terasawa, \\ John V. Priestley and Kimihito Tashima
}

Additional information is available at the end of the chapter

http://dx.doi.org/10.5772/58557

\section{Introduction}

\subsection{Capsaicin-sensitive afferent nerves}

Capsaicin-sensitive afferent nerves play an important role in maintaining the integrity of the gastric mucosa. Stimulation of these nerves by capsaicin has been demonstrated to protect gastric mucosa from a variety of noxious stimuli through increased gastric mucosal blood flow (GMBF) [1, 2]. The binding site of capsaicin has been cloned, and named transient receptor potential vanilloid type 1 (TRPV1), a nonselective cation channel [3]. It is reported that capsaicin stimulates these afferent nerves through activation of TRPV1, resulting in gastric mucosal protection [4] and gastric hyperemic response [5], which are mediated by both calcitonin gene-related peptide (CGRP) and nitric oxide (NO) [6, 7]. Indeed, it has been shown that gastric mucosal vasodilation induced by intragastric application of capsaicin was reduced by the inhibitor of NO synthase (NOS), $N^{\mathrm{G}}$-nitro-L-arginine methyl ester (L-NAME) [8].

\subsection{Nitric oxide synthase}

NO has been reported to affect gastric mucosal blood flow [9]. In mammals, three isoforms of NOS encoded by different genes have been identified [10]. The constitutively expressed isoforms include neuronal NOS (nNOS) present in the neuron and endothelial NOS (eNOS) present in the endothelium lining the vasculature. Inducible NOS (iNOS) requires a stimulus (cytokines and lipopolysaccharides) for expression in specific cell types such as macrophages, 
neutrophils, and epithelial cells. Therefore, eNOS-derived NO is assumed to contribute to the gastric hyperemic response to capsaicin although the gastric mucosa has been shown to contain not only eNOS [11, 12] but also nNOS [11] and iNOS [13].

Interestingly, Chen et al. [14] speculated that NO comes from not only endothelium but also nitroxidergic nerves in the submucosa in gastric vasodilation response to capsaicin. However, it has not been studied which NOS isoforms contribute to gastric hyperemic responses to capsaicin thus far.

\subsection{Aim}

In the present report, we examined to determine the roles of NOS isoforms on gastric hyperemic response to capsaicin in urethane-anesthetized rats by using pharmacological tools, including $N^{5}$-[imino (propylamino) methyl]-L-ornithine, (NPLA; a selective nNOS inhibitor), $N^{5}$-(l-iminoethyl)-L-ornithine (L-NIO; a selective eNOS inhibitor), and 1400W (a selective iNOS inhibitor). In addition, we investigated the localization relationship of nNOS and TRPV1expressing nerves in rat stomachs by using immunohistochemical analysis.

\section{Material and methods}

\subsection{Animals}

Male Sprague-Dawley strain rats (SLC, Hamamatsu, Japan) weighing 180-220 g were used. Animals were housed under controlled environmental conditions (temperature, $24 \pm 2{ }^{\circ} \mathrm{C}$ and lights on 7:00 am to 7:00 pm) and fed commercial rat chow MF (Oriental Yeast, Tokyo, Japan). The animals were kept in individual cages with raised mesh bottoms to prevent coprophagy, and they were deprived of food but allowed free access to tap water for $18 \mathrm{~h}$ before the experiments. Animal experiments were performed according to the "Guiding Principles for the Care and Use of Laboratory Animals" approved by the Japanese Pharmacological Society and the guidelines approved by the Ethical Committee on Animal Care and Animal Experimentation of Josai International University (\#52). The number of animals used was kept to the minimum necessary for meaningful interpretation of the data.

\subsection{Preparations and drugs used}

Atenolol, capsaicin, CMC, dimethyl sulfoxide (DMSO), $d l$-isoproterenol, and urethane (ethyl carbamate) were obtained from Wako Pure Chemical Industries (Osaka, Japan). L-arginine, LNAME, and omeprazole were from Sigma-Aldrich (St. Louis, MO, USA). NPLA and L-NIO came from Tocris Cookson (Ellisville, MO, USA). N-(4-t-Butylphenyl)-4-(3-chlopyridin-2-yl) tetrahydropyrazine-1(2H)-carboxamide (BCTC) was purchased from BIOMOL (Plymouth Meeting, PA, USA). Capsaicin was dissolved in Tween 80 -ethanol solution (10\% ethanol, 10\% Tween 80 , and $80 \%$ saline) for s.c. injection or suspended in $0.5 \%$ CMC for mucosal application. Omeprazole was suspended in $0.5 \%$ CMC for i.p. injection. BCTC was dissolved in DMSO prior to dilution in saline, and the final concentration of DMSO was less than $1.0 \%$. Other drugs 
were dissolved in saline with no organic solvent or detergent. Each drug was prepared immediately before use and was given in a volume of $0.5 \mathrm{ml} / 100 \mathrm{~g}$ of body wt. in the case of i.p. and s.c. administration or in a volume of $0.1 \mathrm{ml} / 100 \mathrm{~g}$ of body wt. in case of i.v. administration. Control animals received the vehicle alone.

\subsection{Gastric mucosal blood flow}

The measurement of gastric blood flow was carried out as described previously [15]. The animals were anesthetized with urethane $(1.25 \mathrm{~g} / \mathrm{kg}$, i.p.). The stomach was exposed through a midline incision, delivered onto the abdominal surface by gentle traction on the spleen, and pylorus was ligated. The lucite chamber was used for maintaining ex vivo conditions of gastric mucosa. The lucite chamber, known as the ex vivo chamber, consists of two parts: one part is a lucite base and the other is plastic rim which has two holes on the side wall. Two holes are cannulated for perfusing the mucosa with saline $\left(154 \mathrm{mmol} / 1 \mathrm{NaCl}, 37^{\circ} \mathrm{C}\right)$ at a flow rate of 1 $\mathrm{ml} / \mathrm{min}$. The lucite base was lowered over the animals, and the stomach was drawn though the center hole with the forceps applied only to the forestomach. The stomach was then opened along the greater curvature from the middle part of the forestomach to the area where the epiploic artery is terminated, and the edges were pinned out by gently stretching the glandular mucosa. The plastic rim was then applied and tightened down on the mucosa. Under these conditions, the exposed area was exclusively the glandular mucosa, mostly consisting of corpus region. The chamber was set at the level of the abdominal wall so that the external wall of the stomach remained inside the abdominal cavity. The body temperature was maintained at a temperature similar to that of the rectum at around $37^{\circ} \mathrm{C}$ by using a small animal warmer and thermometer (Bio Research Center, Model BWT-100, Nagoya, Japan). Gastric mucosal blood flow was measured by laser-Doppler flowmetry (Advance, Model ALF-21N, Tokyo, Japan) and by a non-touching probe (1 $\mathrm{mm}$ in diameter) on the surface of the corpus mucosa. After gastric mucosal blood flow was well stabilized, the perfusion was discontinued, the luminal solution was removed, and then the mucosa was exposed to $2 \mathrm{ml}$ of capsaicin for 10 $\mathrm{min}$. After application, the mucosa was rinsed with saline, another $2 \mathrm{ml}$ of saline was instilled, and the perfusion resumed. Changes in the gastric mucosal blood flow were continuously monitored and recorded for 2-h test periods by using the PowerLab system (Model ML845; AD Instruments, Bella Vista, NSW, Australia).

\subsection{Experimental procedures}

A TRPV1 antagonist $N$-(4-t-butylphenyl)-4-(3-chlopyridin-2-yl) tetrahydropyrazine-1(2H)carboxamide (BCTC) $(0.8 \mathrm{mmol} / \mathrm{L}$, i.g. $)$ [16], a non-selective NOS inhibitor $N^{\mathrm{G}}$-nitro-L-arginine methyl ester (L-NAME; 5 and $10 \mathrm{mg} / \mathrm{kg}$, i.v.), a nNOS selective inhibitor NPLA (0.02 and 0.2 $\mathrm{mg} / \mathrm{kg}$, i.v.), an eNOS selective inhibitor L-NIO (3 and $10 \mathrm{mg} / \mathrm{kg}$, i.v.), and an iNOS selective inhibitor $1400 \mathrm{~W}$ ( 3 and $10 \mathrm{mg} / \mathrm{kg}$, i.v.) were administered $20 \mathrm{~min}$ before exposing the stomach to $2 \mathrm{ml}$ of capsaicin for $10 \mathrm{~min}$. In one group, L-arginine (300 mg/kg, i.v.) was administered twice $40 \mathrm{~min}$ and $60 \mathrm{~min}$ before the application of capsaicin. 


\subsection{Chemical deafferentation}

Chemical deafferentation was performed two weeks before the experiment by successive injections of capsaicin subcutaneously once daily for $3 \mathrm{~d}(20,30$, and $50 \mathrm{mg} / \mathrm{kg})$ [17]. All capsaicin injections were performed under ether anesthesia, and the rats were pretreated with the beta-adrenergic receptor agonist isoproterenol $(0.01 \mathrm{mg} / \mathrm{kg}, \mathrm{i} . \mathrm{m}$. $)$ and the selective beta 1adrenergic antagonist atenolol $(0.01 \mathrm{mg} / \mathrm{kg}$, i.m. $)$ to counteract the respiratory impairment associated with capsaicin injection. To check for the effectiveness of the treatment, a drop of a $0.1 \mathrm{mg} / \mathrm{ml}$ solution of capsaicin in $0.5 \%$ carboxymethyl cellulose solution (CMC) was instilled into one eye of each rat, and the protective wiping movements were counted [18].

\subsection{Immunohistochemical study}

The immunohistochemical procedures were performed as described previously [4]. The corpora of rat stomachs were fixed by immersion in fresh $4 \%$ paraformaldehyde in $0.1 \mathrm{~mol} / \mathrm{l}$ phosphate buffer ( $\mathrm{pH} 7.4)$ for $2 \mathrm{~h}$ at $4^{\circ} \mathrm{C}$, and washed three times with phosphate-buffered saline (PBS). They were cryoprotected overnight in $0.1 \mathrm{~mol} / \mathrm{l}$ phosphate buffer containing $20 \%$ sucrose. The tissues were frozen in OCT mounting medium (Sakura Finetek Europe, Zoeterwoude, The Netherlands), and sectioned on a cryostat (Leica, Hemel Hempstead, UK) at a thickness of $60 \mu \mathrm{m}$. The tissue sections were thaw-mounted onto Superfrost Plus slides (BDH Laboratory Supplies, Poole, UK). Prior to staining, the slide-mounted sections were incubated in PBS containing $0.3 \%$ hydrogen peroxide for $30 \mathrm{~min}$ to quench endogenous peroxidase activity and washed with PBS. The sections were successively incubated in $10 \%$ normal donkey serum containing $0.2 \%$ Triton $\mathrm{X}-100,0.1 \%$ sodium azide in PBS for $1 \mathrm{~h}$, and polyclonal antiTRPV1 antibody (1:15000) for $40 \mathrm{~h}$ at room temperature. TRPV1 immunoreactivity was detected using a polyclonal antiserum raised in rabbit against the carboxy terminus of rat TRPV1 provided by Dr. David Julius. After washes in PBS, the sections were incubated with biotinylated donkey anti-rabbit immounoglobulin G (1:400; Jackson Immunoresearch Laboratories, West Grove, PA, USA) for $90 \mathrm{~min}$ at room temperature. After further washes, the sections were incubated in stretavidin biotin-peroxidase complex (1:5 in PBS; Vectastain Elite ABC kit, Vector Laboratories, Peterborough, UK) for $1 \mathrm{~h}$ at room temperature followed by fluorescein tyramide (1:75; TSA kit, PerkinElmer Life Sciences, Boston, MA, USA). In control experiments, the TRPV1 antibody was omitted from the staining procedures to verify the specificity of the staining. No immunolabeling was observed in these controls.

Double staining of the section with TRPV1 antiserum combined with protein gene product 9.5 (PGP 9.5) and nNOS antisera was also performed. TRPV1 staining was carried out first using the TSA procedure with FITC, following by PGP 9.5 or nNOS staining using indirect labeling procedure as described below. The sections were incubated for $40 \mathrm{~h}$ at room temperature in the PGP 9.5 or nNOS primary antibody solution, and then washed three times for $10 \mathrm{~min}$ each in PBS. To visualize the labeling, sections were then incubated for $4 \mathrm{~h}$ with the corresponding secondary antibody linked to tetramethyl rhodamine isothiocyanate (TRITC, 1:400; Jackson Immunoresearch Laboratories). Antisera were diluted in PBS containing $0.2 \%$ Triton X-100 and $0.1 \%$ sodium azide. Two sets of controls were processed for this double labeling. First, the primary antibodies in the first and second stainings were omitted from the staining procedures. 
No immunolabeling was observed in this control. Second, only the primary antibody in the second staining was omitted from the staining procedures. In this control, FITC labeling of TRPV1 fibers was seen, but TRITC labeling was not.

\subsection{Microscopy}

Sections were viewed on either a Leica epifluorescence microscope using Y3 (TRITC) and L4 (FITC) filter blocks, or on a upright Zeiss confocal laser scanning microscope system (LSM510) and $\times 20, \times 40$ Plan Neofluar objectives. On the latter system, single tracking mode was used for single FITC labeling. Sections were scanned in multitracking mode (to avoid channel cross talk) when double labeling was done with FITC (488 nm) and TRITC (543 nm). Each image was produced by projecting all the slices of the stack onto one plane. Final plates were prepared using Adobe Photoshop.

\subsection{Statistics}

The data are presented as the means \pm S.E.M. from 3-7 rats per group. The statistical significance of differences between two groups was assessed using Student's $t$-test. Multiple comparisons against a single control group were made by a one-way analysis of variance ANOVA with Bonferroni correction. The level of significance was set at 0.05. Sigma Stat 3.1 software (Jandel Scientific Software, San Rafael, CA, USA) procedure was applied for statistical analysis.

\section{Results}

\subsection{Effects of intragastric capsaicin on gastric mucosal blood flow}

Intragastric capsaicin $(0.33,1$, and $3.3 \mathrm{mmol} / \mathrm{l})$ induced gastric hyperemic responses in a concentration-dependent manner: a significant effect was observed at concentrations greater than $1 \mathrm{mmol} / \mathrm{l}$ (Fig. 1B). The response of gastric mucosal blood flow to capsaicin $(0.33,1$, and $3.3 \mathrm{mmol} / \mathrm{l}$ ) during intragastric application for $10 \mathrm{~min}$ was $121.5 \pm 2.6 \%, 146.4 \pm 8.9 \%$, and 178.6 $\pm 12.9 \%$, respectively. Interestingly, gastric mucosal blood flow in response to $3.3 \mathrm{mmol} / \mathrm{l}$ capsaicin remained significantly high despite the removal of capsaicin from the chamber (Fig. 1A). At 20 and 50 minutes after removal of capsaicin, gastric mucosal blood flow showed a persistent increase (about $\sim 120-130 \%$ ) as compared to pre-application values (Fig. 1C). Mucosal application of the control solution ( $0.5 \% \mathrm{CMC})$ did not increase gastric mucosal blood flow. Since the effect of capsaicin on gastric mucosal blood flow reached the maximum values during the application and remained significantly elevated after removal of capsaicin at 3.3 $\mathrm{mmol} / \mathrm{l}$, this concentration was used in the following experiments for examining the effects of various agents on the gastric mucosal blood flow in response to capsaicin. 
A
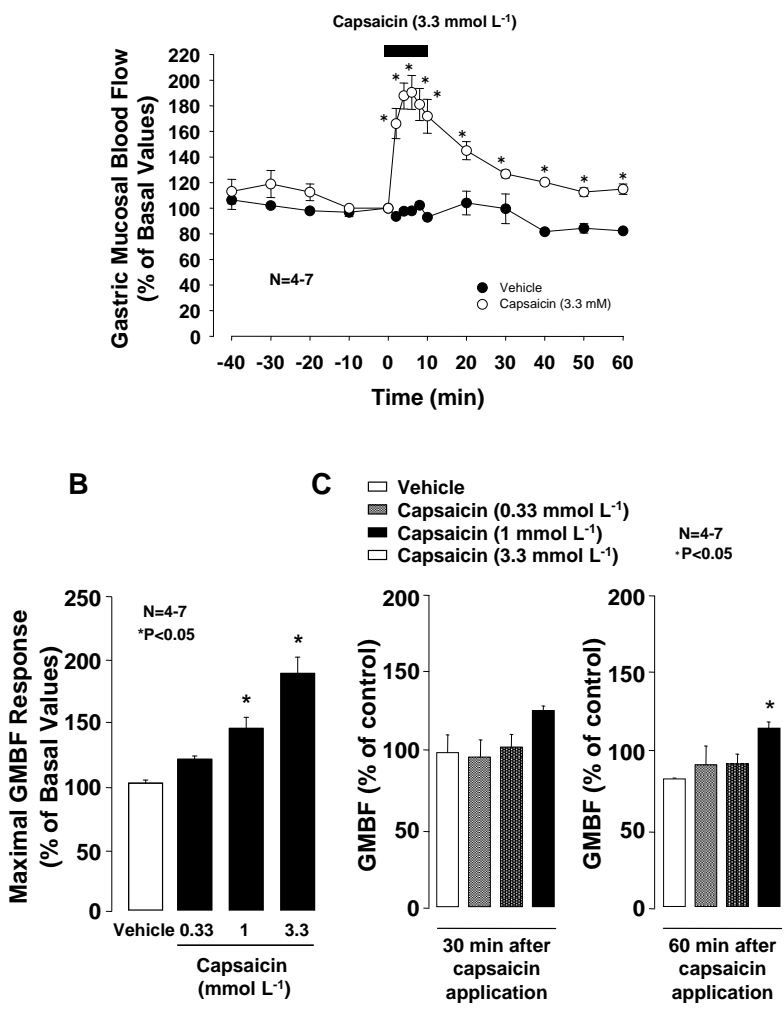

Figure 1. Effect of mucosal application of capsaicin on gastric mucosal blood flow (GMBF) in the ex vivo stomach of anesthetized rats. Figure A shows the time course of analysis for GMBF in response to capsaicin (3.3 mmol/I) in anesthetized rats. Capsaicin was topically applied to the mucosa for $10 \mathrm{~min}$ from time 0 , and the stomach was perfused with saline before and after the application. The data are expressed as a \% increase of baseline values and represent the means \pm S.E. of values obtained every 2 or 10 min from 4 to 7 rats. * indicates statistically significant difference at $\mathrm{P}<0.05$ compared to the group treated with control $(0.5 \% \mathrm{CMC})$. Figure B shows the maximum GMBF response during capsaicin (0.33-3.3 mmol/l) application, and figure C shows the GMBF response at 20 and 50 min after removal of capsaicin (0.33-3.3 mmol/l). The data are expressed as a \% increase of baseline values, and represent the means \pm S.E. from 4 to 7 rats. * indicates statistically significant difference at $\mathrm{P}<0.05$ compared to the group treated with control $(0.5 \% \mathrm{CMC})$. Note that GMBF in response to capsaicin $(3.3 \mathrm{mmol} / \mathrm{l})$ was significantly increased during application, and remained moderately elevated even after removal of capsaicin from the chamber.

The increased gastric mucosal blood flow in animals in response to capsaicin ( $3.3 \mathrm{mmol} / \mathrm{l})$ was totally abolished in the animals when the mucosa was exposed to a TRPV1 antagonist BCTC ( $0.8 \mathrm{mmol} / \mathrm{l})$ (Fig. 2). The maximum response of gastric mucosal blood flow induced by 3.3 $\mathrm{mmol} / \mathrm{l}$ capsaicin in the presence of BCTC was $102.4 \pm 6.1 \%$ throughout the experiment. A similar phenomenon was observed in the animals following chemical deafferentation by consecutive injections of capsaicin (neurotoxic dose: total $100 \mathrm{mg} / \mathrm{kg}$, s.c.) at two weeks before the experiment (Fig. 2). In those animals, the gastric mucosal blood flow remained unchanged 
during and after exposure of the mucosa to capsaicin $(3.3 \mathrm{mmol} / \mathrm{l})$, the maximum response was $103.1 \pm 4.2 \%$. It was found that intragastric capsaicin produced a significant and persistent increase in gastric mucosal blood flow via activation of TRPV1 expressed in capsaicin-sensitive sensory nerves in anesthetized rats.

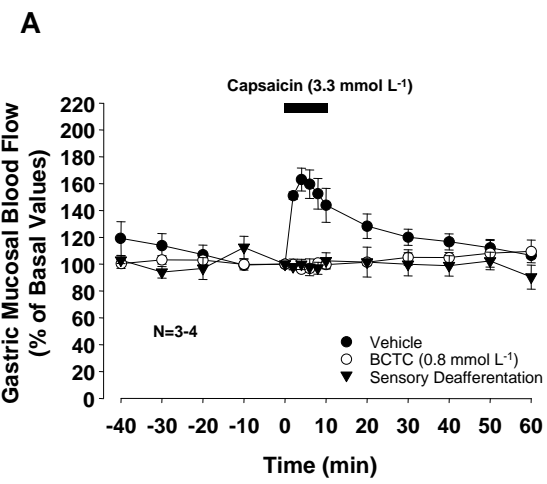

B

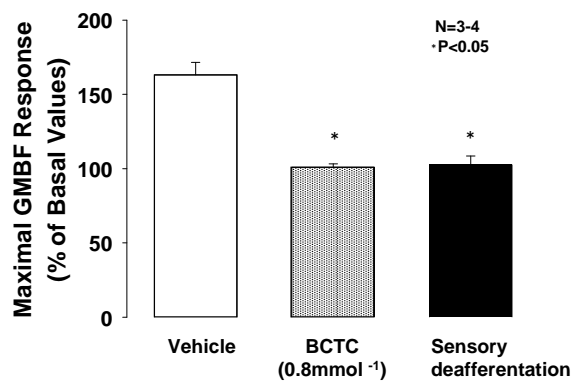

Figure 2. Effects of BCTC, a TRPV1 antagonist, and sensory deafferentation on gastric mucosal blood flow (GMBF) induced by mucosal application of capsaicin in the ex vivo stomach of anesthetized rats. Figure A shows the time course of analysis for GMBF response to capsaicin $(3.3 \mathrm{mmol} / \mathrm{l})$ in anesthetized rats treated with BCTC or sensory deafferentation. Capsaicin was topically applied to the mucosa for $10 \mathrm{~min}$ and the stomach was perfused with saline before and after the application. BCTC $(0.8 \mathrm{mmol} / \mathrm{l})$ was applied to the chamber for $30 \mathrm{~min}$, starting at $20 \mathrm{~min}$ before the capsaicin application. Chemical deafferentation (capsaicin pretreatment) was performed 2 weeks before the experiment by consecutive injections of capsaicin s.c. once daily for 3 days (total dose: $100 \mathrm{mg} / \mathrm{kg}$ ). The data are expressed as a \% increase of baseline values and represent the mean \pm S.E. of values obtained every 2 or 10 min from 3 to 4 rats. * indicates statistically significant difference at $\mathrm{P}<0.05$ compared to the group treated with vehicle. Figure $\mathrm{B}$ shows a maximum response of GMBF induced by mucosal application of capsaicin ( $3.3 \mathrm{mmol} / \mathrm{l})$. The data are expressed as a $\%$ increase of baseline values and represent the mean \pm S.E. from 3 to 4 rats. * indicates statistically significant difference at $\mathrm{P}<0.05$ compared to the group treated with vehicle. Note that increased GMBF in response to capsaicin was completely abolished by BCTC and sensory deafferentation. 


\subsection{Effects of L-NAME, a non-selective NOS inhibitor, and combined treatment with L- arginine on gastric mucosal blood flow in response to capsaicin}

Gastric mucosal blood flow was temporarily increased after the injection of L-NAME was injected but immediately returned to the baseline value (Fig. 3A). The increase in gastric mucosal blood flow in response to capsaicin $(3.3 \mathrm{mmol} / \mathrm{l})$ seen in control rats apparently attenuated by L-NAME in a dose-dependent manner. The maximum response of gastric mucosal blood flow in response to capsaicin in animals treated with L-NAME (5 and 10 $\mathrm{mg} / \mathrm{kg}$ ) was $138.8 \pm 11.6 \%$ and $125.6 \pm 11.6 \%$, respectively (Fig.3B). The administration of 300 $\mathrm{mg} / \mathrm{kg}$ L-arginine, a substrate for NOS, twice reversed the inhibitory effects of L-NAME (10 $\mathrm{mg} / \mathrm{kg}$ ) on gastric hyperemic response both during and after removal of capsaicin (Fig. 3). Thus, gastric hyperemia in response to capsaicin was attributable to both NO-dependent hyperemia during the application, followed by NO-dependent hyperemia alone after removal of capsaicin in anesthetized rats.

A
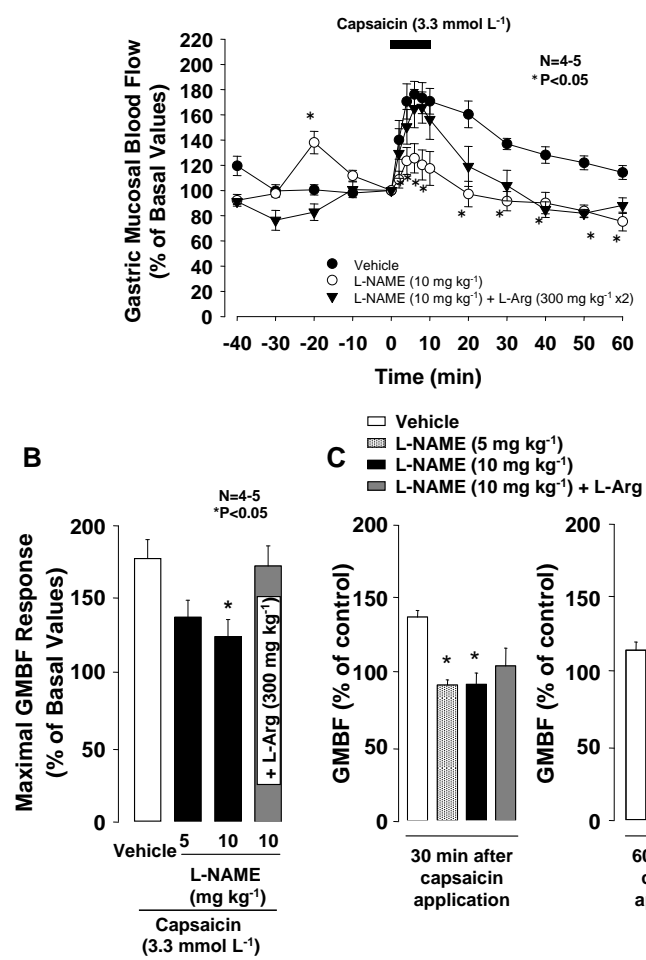

$\begin{array}{ll} & \square \text { Vehicle } \\ C \quad \square & \text { L-NAME }\left(5 \mathrm{mg} \mathrm{kg}^{-1}\right) \\ \text { L-NAME }\left(10 \mathrm{mg} \mathrm{kg}^{-1}\right)\end{array}$

$\square$ L-NAME $\left(10 \mathrm{mg} \mathrm{kg}^{-1}\right)+$ L-Arg $\left(300 \mathrm{mg} \mathrm{kg}^{-1} \times 2\right)$

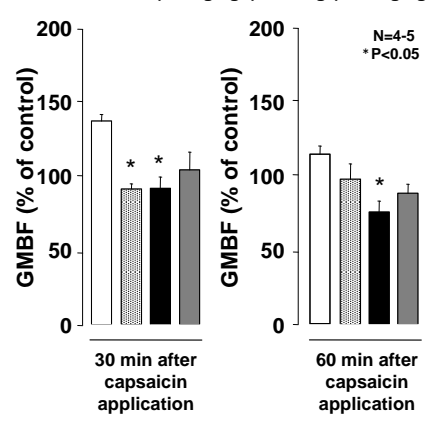

Figure 3. Effects of L-NAME, a non-selective NOS inhibitor, and combined treatment with L-arginine $(300 \mathrm{mg} / \mathrm{kg}, \mathrm{x} 2)$ on gastric mucosal blood flow (GMBF) induced by mucosal application of capsaicin in the ex vivo stomach of anesthetized rats. Figure A shows the time course of analysis for GMBF response to capsaicin (3.3 mmol/l) in anesthetized rats 
pretreated with L-NAMA and L-arginine. Capsaicin $(3.3 \mathrm{mmol} / \mathrm{l})$ was topically applied to the mucosa for $10 \mathrm{~min}$ and the stomach was perfused with saline before and after the application. L-NAME ( 5 and $10 \mathrm{mg} / \mathrm{kg}$ ) was given via intravenous injection $20 \mathrm{~min}$ before capsaicin application. L-NAME by itself showed the temporary increase of GMBF when was injected, but immediately returned to baseline value. L-arginine $(300 \mathrm{mg} / \mathrm{kg})$ was given via intravenous injection 40 and $60 \mathrm{~min}$ before capsaicin application. The data are expressed as a \% increase of baseline values and represent the means \pm S.E. of values obtained every 2 or 10 min from 4 to 5 rats. * indicates statistically significant difference at $\mathrm{P}<0.05$ compared to the group treated with vehicle (saline). Figure $\mathrm{B}$ shows the maximum response of GMBF during capsaicin $(3.3 \mathrm{mmol} / \mathrm{l})$ application in animals with L-NAME $(5$ and $10 \mathrm{mg} / \mathrm{kg})$ or L-NAME $(10 \mathrm{mg} / \mathrm{kg})$ plus L-arginine (300 mg/kg, x2). Figure C shows a response of GMBF at 20 and $50 \mathrm{~min}$ after removal of capsaicin (3.3 mmol/l) with LNAME (5 and $10 \mathrm{mg} / \mathrm{kg}$ ) or L-NAME $(10 \mathrm{mg} / \mathrm{kg})$ plus L-arginine $(300 \mathrm{mg} / \mathrm{kg}, \times 2)$. The data are expressed as a $\%$ increase of baseline values and represent the mean \pm S.E. from 4 to 5 rats. * indicates statistically significant difference at $\mathrm{P}<0.05$ compared to the group treated with vehicle (saline). Note that increased GMBF during and after removal of capsaicin was significantly inhibited by L-NAME $(10 \mathrm{mg} / \mathrm{kg})$, whose response was reversed by pretreatment with Larginine (300 mg/kg, x2).

\subsection{Effect of NPLA, a selective nNOS inhibitor, on gastric mucosal blood flow in response to capsaicin}

The roles of different isoforms of NOS in the gastric hyperemia induced by capsaicin were investigated by using selective NOS inhibitors including a nNOS inhibitor NPLA, an eNOS inhibitor L-NIO, and an iNOS inhibitor 1400W. Administration of the nNOS-specific inhibitor NPLA alone did not significantly affect gastric mucosal blood flow (Fig. 4A). Interestingly, the increase in gastric mucosal blood flow in response to capsaicin $(3.3 \mathrm{mmol} / \mathrm{l})$ during the application, but not after removal, was apparently decreased by $0.2 \mathrm{mg} / \mathrm{kg}$ NPLA. The maximum responses of gastric mucosal blood flow during capsaicin application in animals treated with NPLA (0.02 and $0.2 \mathrm{mg} / \mathrm{kg}$ ) were $182.1 \pm 26.6 \%$ and $141.9 \pm 8.9 \%$, respectively (Fig. $4 \mathrm{~B})$. In contrast, the persistent increase in gastric mucosal blood flow after removal of capsaicin was not affected by NPLA $(0.2 \mathrm{mg} / \mathrm{kg})$, with the responses being $138.3 \pm 17.3 \%$ and $126.5 \pm$ $15.1 \%$, respectively (Fig. 4 C).

\subsection{Effect of L-NIO, a selective eNOS inhibitor, on gastric mucosal blood flow in response to capsaicin}

Administration of the selective eNOS inhibitor L-NIO $(10 \mathrm{mg} / \mathrm{kg})$ by itself temporarily increased gastric mucosal blood flow, but the gastric mucosal blood flow immediately returned to the baseline value (Fig. 5A). The gastric hyperemic response after treatment with capsaicin during the application was not significantly affected by L-NIO ( 3 and $10 \mathrm{mg} / \mathrm{kg}$ ), and the maximum responses being $158.7 \pm 8.4 \%$ and $160.0 \pm 9.6 \%$, respectively (Fig. 5B). However, the persistent increase in gastric mucosal blood flow after removal of capsaicin was obviously attenuated by L-NIO (10 mg/kg) (Fig. 5A, C). In treatment with L-NIO (10 mg/kg), the gastric mucosal blood flow responses at 20 and $50 \mathrm{~min}$ after removal of capsaicin were $96.2 \pm 7.2 \%$ and $89.5 \pm 6.2 \%$, respectively. 
A
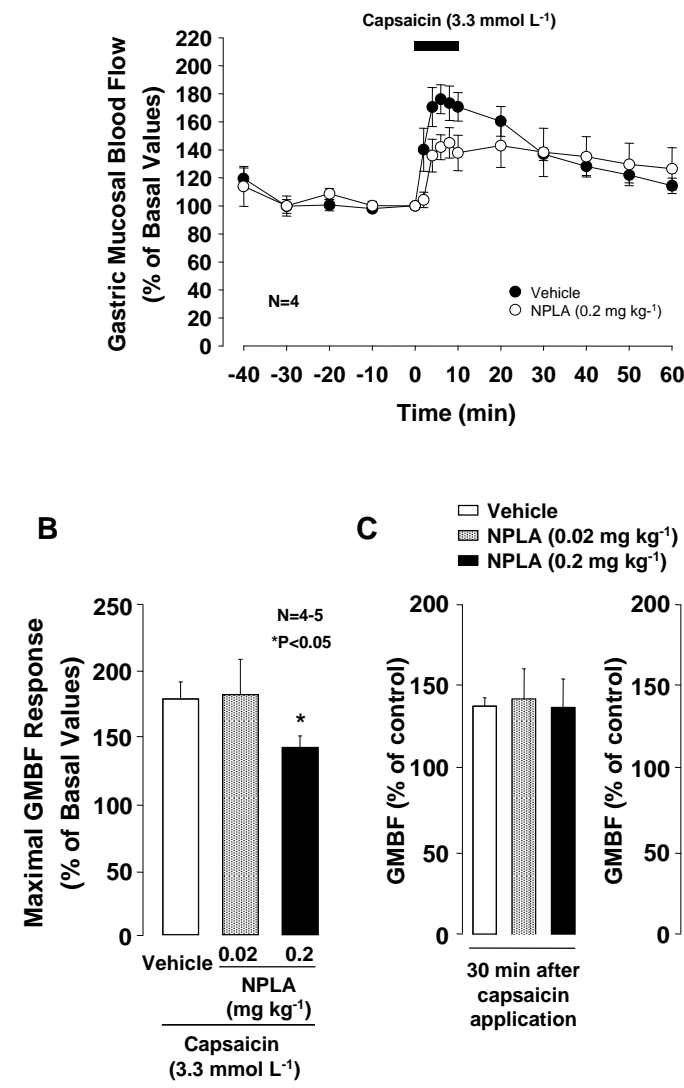

C $\square$ Vehicle

C NPLA $\left(0.02 \mathrm{mg} \mathrm{kg}^{-1}\right)$

NPLA $\left(0.2 \mathrm{mg} \mathrm{kg}^{-1}\right) \quad \mathrm{N}=4-5$
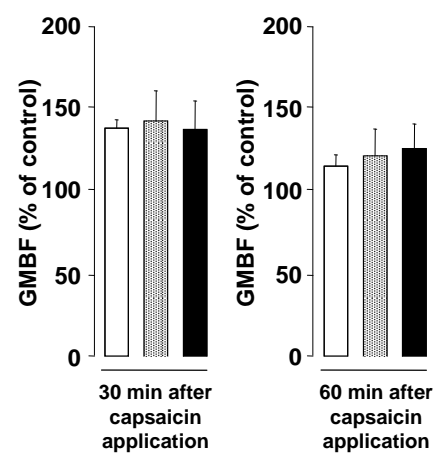

Figure 4. Effect of NPLA, a selective neuronal NOS inhibitor, on gastric mucosal blood flow (GMBF) induced by capsai$\mathrm{cin}$ in the ex vivo stomach of anesthetized rats. Figure A shows the time course of the effect of NPLA $(0.2 \mathrm{mg} / \mathrm{kg}) \mathrm{on}$ GMBF response to capsaicin $(3.3 \mathrm{mmol} / \mathrm{l})$ in anesthetized rats. Capsaicin $(3.3 \mathrm{mmol} / \mathrm{l})$ was topically applied to the mucosa for $10 \mathrm{~min}$ and the stomach was perfused with saline before and after the application. NPLA $(0.2 \mathrm{mg} / \mathrm{kg}) \mathrm{was}$ given via intravenous injection 20 min before capsaicin application. The data are expressed as a \% increase of baseline values and represent the means \pm S.E. of values obtained every 2 or 10 min from 4 rats. * indicates statistically significant difference at $\mathrm{P}<0.05$ compared to the group treated with vehicle (saline). Figure $\mathrm{B}$ shows the maximum response of GMBF response during capsaicin $(3.3 \mathrm{mmol} / \mathrm{l})$ application in animals with NPLA (0.02 and $0.2 \mathrm{mg} / \mathrm{kg})$. Figure C shows a response of GMBF at 20 and $50 \mathrm{~min}$ after removal of capsaicin ( $3.3 \mathrm{mmol} / \mathrm{l})$ with NPLA $(0.02 \mathrm{and} 0.2 \mathrm{mg} / \mathrm{kg})$. The data are expressed as a \% increase of baseline values and represent the means \pm SE from 4 to 5 rats. * indicates statistically significant difference at $\mathrm{P}<0.05$ compared to the group treated with vehicle (saline). Note that increased GMBF during capsaicin application was inhibited by NPLA, yet persistent increase in GMBF after removal of capsaicin was not affected by NPLA. 
A
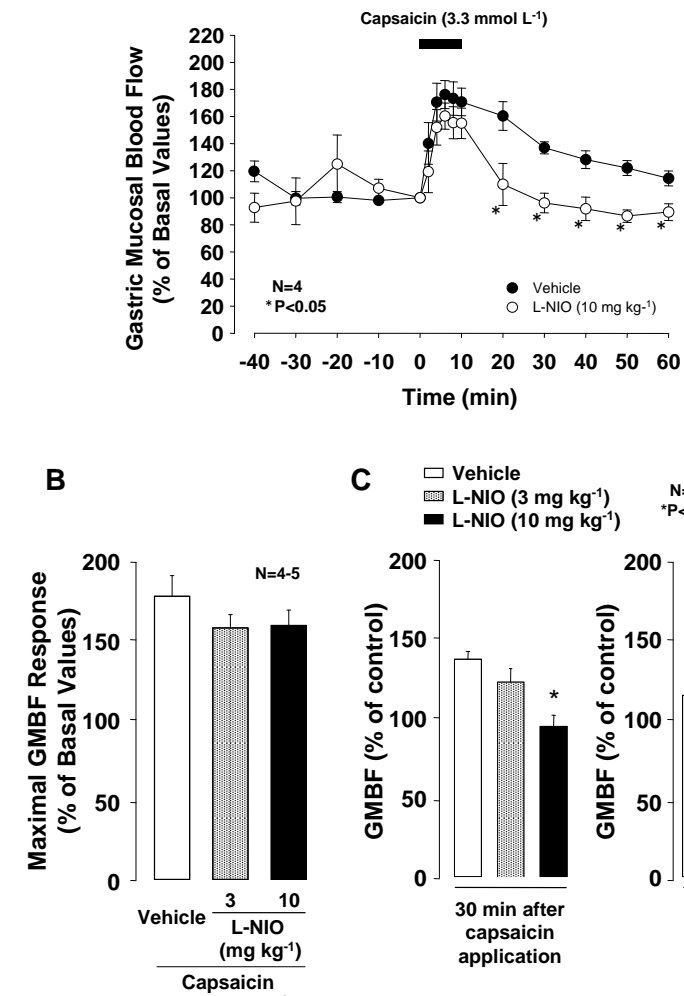

C $\square$ Vehicle

$\begin{array}{lr}\mathrm{N}=4-5 \\ \text { L-NIO }\left(3 \mathrm{mg} \mathrm{kg}^{-1}\right) & { }^{\mathrm{P}}<0.05\end{array}$
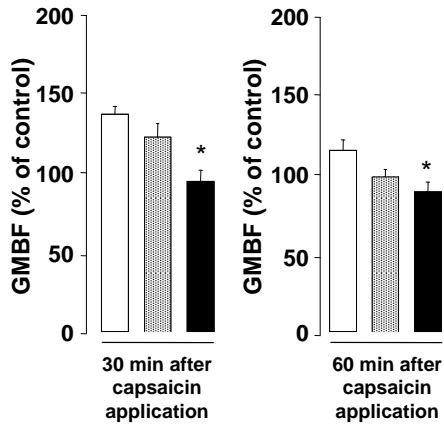

(3.3 $\left.\mathrm{mmol} \mathrm{L}^{-1}\right)$

Figure 5. Effect of L-NIO, a selective endothelial NOS inhibitor, on gastric mucosal blood flow (GMBF) induced by capsaicin in the ex vivo stomach of anesthetized rats. Figure A shows the time course of analysis for the effect of L-NIO (10 $\mathrm{mg} / \mathrm{kg}$ ) on GMBF response to capsaicin $(3.3 \mathrm{mmol} / \mathrm{l})$ in anesthetized rats. Capsaicin ( $3.3 \mathrm{mmol} / \mathrm{l})$ was applied topically to the mucosa for $10 \mathrm{~min}$ and the stomach was perfused with saline before and after the application. L-NIO (10 $\mathrm{mg} / \mathrm{kg}$ ) was injected intravenously $20 \mathrm{~min}$ before capsaicin application. L-NIO by itself induced a temporary increase of GMBF when injected, but it immediately returned to baseline value. The data are expressed as a \% increase of baseline values and represent the means \pm S.E. of values obtained every 2 or 10 min from 4 rats. * indicates statistically significant difference at $\mathrm{P}<0.05$ compared to the group treated with vehicle (saline). Figure $\mathrm{B}$ shows the maximum response of GMBF response during capsaicin ( $3.3 \mathrm{mmol} / \mathrm{l}$ ) application in animals with L-NIO (3 and $10 \mathrm{mg} / \mathrm{kg}$ ). Figure C shows the response of GMBF at 20 and 50 min after removal of capsaicin $(3.3 \mathrm{mmol} / \mathrm{l})$ with L-NIO (3 and $10 \mathrm{mg} / \mathrm{kg})$. The data are expressed as a \% increase of baseline values and represent the means \pm S.E. from $4-5$ rats. * indicates statistically significant difference at $\mathrm{P}<0.05$ compared to the group treated with the vehicle (saline). Note that L-NIO significantly inhibited persistent increase of GMBF after removal of capsaicin, although GMBF response during capsaicin application was not affected by L-NIO. 


\subsection{Effect of $1400 \mathrm{~W}$, a selective iNOS inhibitor, on gastric mucosal blood flow in response to capsaicin}

Gastric mucosal blood flow remained unchanged by the administration of an iNOS selective inhibitor 1400W (10 mg/kg) (Fig. 6). In addition, the increased gastric mucosal blood flow I response to capsaicin was not also affected by $1400 \mathrm{~W}$ ( 3 and $10 \mathrm{mg} / \mathrm{kg}$ ) (Fig. 6).

A

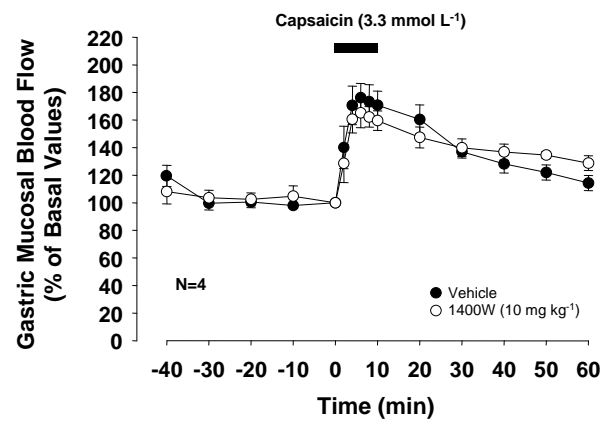

B

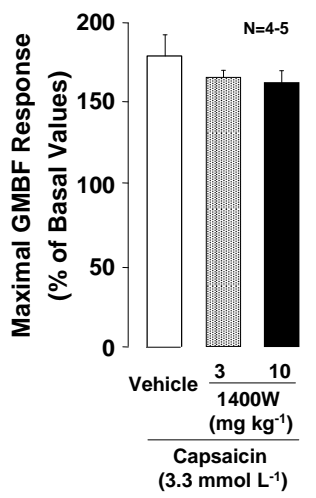

C $\square$ Vehicle

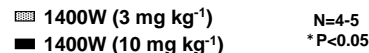
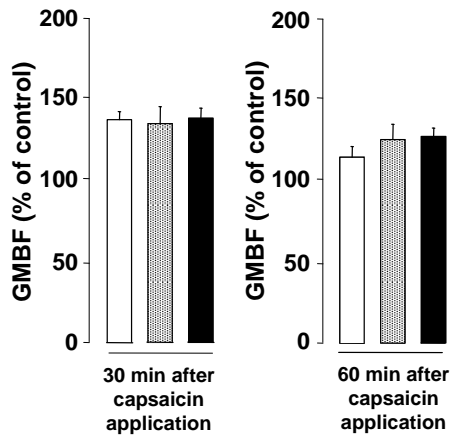

Figure 6. Effect of 1400W, a selective inducible NOS inhibitor, on gastric mucosal blood flow (GMBF) induced by capsaicin in the ex vivo stomach of anesthetized rats. Figure A shows the time course of analysis for the effect of $1400 \mathrm{~W}$ $(10 \mathrm{mg} / \mathrm{kg})$ on GMBF response to capsaicin $(3.3 \mathrm{mmol} / \mathrm{l})$ in anesthetized rats. Capsaicin (3.3 mmol/l) was topically applied to the mucosa for $10 \mathrm{~min}$ and the stomach was perfused with saline before and after the application. $1400 \mathrm{~W}$ ( 3 and $10 \mathrm{mg} / \mathrm{kg}$ ) was given via intravenous injection $20 \mathrm{~min}$ before capsaicin application. The data are expressed as a \% increase of baseline values and represent the means \pm S.E. of values obtained every 2 or 10 min from 4 rats. Figure B shows the maximum response of GMBF response during capsaicin $(3.3 \mathrm{mmol} / \mathrm{l})$ application in animals treated with 1400W (3 and $10 \mathrm{mg} / \mathrm{kg}$ ). Figure C shows the responses of GMBF (3.3 mmol/l) at 20 and 50 min after removal of capsaicin. The data are expressed as a \% increase of baseline values and represent the mean \pm S.E. from 4 to 5 rats. Note that increased GMBF in response to capsaicin was not affected by $1400 \mathrm{~W}$. 


\subsection{TRPV1-immunoreactivity in corpus and antrum of stomach}

Extensive TRPV1-immunoreactivity was detected in fundus, corpus and antrum of the stomach [4, 19]. Immunoreactivity was present in all layers of the corpus and in all cases appeared axon-like in nature (Fig. 7A). This identification was confirmed by double labeling with the pan axonal marker PGP 9.5, which confirmed that all TRPV1 immunoreactive structures were also PGP 9.5 immunoreactive (Figure 7C). However, not all PGP 9.5-immunoreactive fibers were TRPV1-immunoreactive, reflecting the fact that only a subpopulation of neural elements in the stomach expresses TRPV1. TRPV1 immunoreactivity was also not seen in the PGP 9.5 immunoreactive cell bodies of the myenteric plexus or submucosal plexus. In control experiments, TRPV1-immunoreactivity was not observed in any corpus sections processed without the primary antibody. Similar observation was obtained in all layers of antrum of the stomach [4].

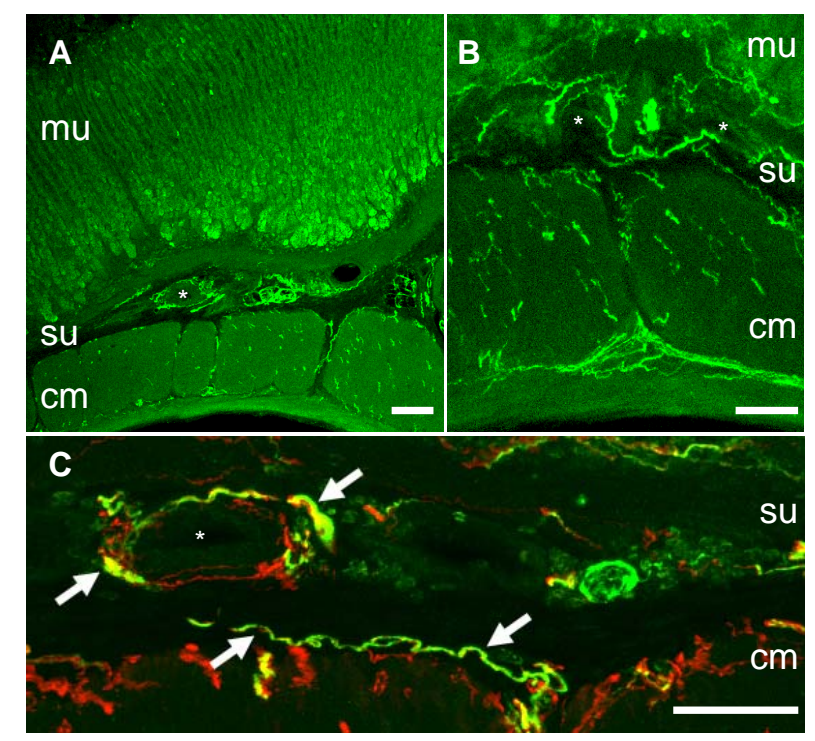

Figure 7. Distribution of TRPV1-immunoreactivity in corpus of rat stomach. mu: mucosa; su: submucosa; cm: circular muscle. (A) A low magnification image showing that TRPV1-immunoractivity is present in all layers of corpus. Asterisk indicates a blood vessel. (B) A high magnification image showing that TRPV1-immunoractivity is present in submucosal and muscular layers of corpus. TRPV1-immunoreactive fibers are observed associated with small blood vessels (asterisk) or along and in the lamina muscularis mucosae. Numerous TRPV1-immunoreactive fibers are found around blood vessels (asterisks) in the submucosal layer. TRPV1-immunoreactive axons are sparse in longitudinal smooth muscle but isolated varicose fibers are present. Fibers are abundant in circular muscle layer where they run parallel to muscle fibers. A large number of nerve fibers are seen in circular smooth muscle layer and in the myenteric nerve plexus. (C) Co-localization of TRPV1-positive neurons with protein gene product 9.5 (PGP 9.5) in corpus submucosa. Rat stomach sections were dual-labelled with TRPV1 in green and PGP 9.5 in red. Merged image is shown in (C). Arrows indicate the co-localization of TRPV1 immunoreactivity with PGP 9.5 immunoreactivity. All TRPV1 immunoreactive structures are PGP 9.5 immunoreactive. Note that in circular smooth muscle, the TRPV1 immunoreactive axons represent a relatively small population of the total population of PGP 9.5 immunoreactive axons. Scale bars are $100 \mu \mathrm{m}$ in (A) and $50 \mu \mathrm{m}$ in (B), (C). 
The TRPV1-immunoreactive varicose fibers were present in the mucosa along gastric glands of corpus and antrum, although Ward et al. (2003) did not observe TRPV1 immunoreactive fibers that penetrate the mucosa [20]. A few TRPV1-immunoreactive varicose nerve fibers were observed in the lamina propria, mainly in association with small vessels or along the muscularis mucosae of antrum and corpus [4, 19]. Numerous TRPV1-immunoreactive axons were also found around arterioles in the submucosal layer. A dense TRPV1-innervation of the submucosal blood vessels was seen running along the major arterioles (Fig. 7B). Occasionally fibers formed baskets around the vessels [4].

\subsection{Coexistence of TRPV1 and nNOS}

Many TRPV1 axons were also observed to be immunoreactive for nNOS (Figure 8). A few axons of this type were present in the mucosa and smooth muscle layers but were particularly abundant in the vicinity of blood vessels in the submucosa [21]. Numerous TRPV1-immunoreactive axons were found around arterioles in the mucosal and submucosal layer.

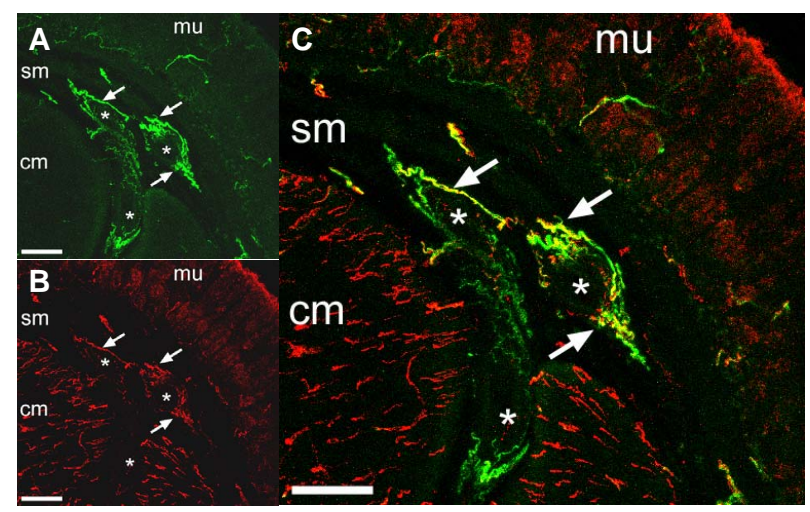

Figure 8. Confocal images showing co-localization of TRPV1-and neuronal NOS-immnuoreactivities in corpus submucosa and muscle. Labeling for TRPV1 (A: green) and neuronal NOS (B: red) is shown separately, and merged in (C). Double immunolabeling for TRPV1 and neuronal NOS demonstrated co-localization in many but not all axons. This photo shows abundant TRPV1 axons containing neuronal NOS in the submucosa, with a high density of immounoreactive axons around blood vessels (asterisk). mu: mucosa; sm: submucosa; cm: circular muscle. Scale bars is $100 \mu \mathrm{m}$.

\section{Discussion}

This report showed that intragastric application of capsaicin facilitates an initial increase in gastric mucosal blood flow by NO mainly derived from nNOS after stimulating capsaicinsensitive sensory nerves, and also the persistent increase in gastric mucosal blood flow after removal of capsaicin is attributed to eNOS/NO in the rat stomach.

Topical application of capsaicin causes dilatation of the submucosal and mucosal arterioles in the rat stomach $[22,23]$, but it has not been fully elucidated how capsaicin really activates 
primary afferent vasodilator neurons in the stomach. Primary afferent neurons express TRPV 1 channels, which mediates the gastrointestinal vasodilator effect of capsaicin. By the use of the TRPV1 blocker BCTC, it was found that this TRPV1 channels appear to participate in the capsaicin-induced hyperemia in the rat stomach [21]. Nerve-selective ablation of capsaicinsensitive afferent neurons has revealed that the mucosal hyperemic response to local capsaicin administration in the rat stomach is mediated by primary afferent vasodilator neurons that originate in the dorsal root ganglia. It was inferred that the capsaicin-induced increase of blood flow is relayed entirely by a peripheral reflex circuit via the TRPV1 activation. The gastric hyperemic response to capsaicin results from an axon reflex between mucosal and submucosal collaterals of afferent neurons [24]. Evidence for an axon reflex-type innervation has been found in submucosal arterioles of the guinea pig ileum in which focal application of capsaicin evokes vasodilatation that spreads beyond the application site [25].

It is known that $\mathrm{NO}$, the endothelium-derived vasodilator, plays an important role in the regulation of the resting gastric mucosal microcirculation [26, 27]. Indeed, an increase in both systemic arterial blood pressure and gastric mucosal blood flow was observed under physiological conditions in rats after a bolus administration of L-NAME.

The gastric mucosal blood flow responses to capsaicin were markedly inhibited by a nonselective NOS inhibitor L-NAME, which indicated that NO is mainly involved in those hyperemic responses. However, L-NAME did not completely inhibit the increases in gastric mucosal blood flow as much as BCTC. This result suggests that gastric mucosal hyperemia in response to capsaicin is attributable not only to $\mathrm{NO}$ but also to other mediators including CGRP and prostaglandins (PGs). Several studies have shown that endogenous PGs, contributes to gastric hyperemic responses to capsaicin by sensitizing TRPV1-expressing afferent nerves, which was revealed by the treatment of indomethacin $(5 \mathrm{mg} / \mathrm{kg}$, s.c.) and the animals lacking IP receptors [28]. In addition, Chen et al. reported the interaction of NO and CGRP in gastric vasodilation through sensory nerves [14]. Our results can be explained on the basis of the previous findings that BCTC functionally antagonizes the action of capsaicin at the peripheral terminals of sensory nerves and inhibits the release of some transmitters such as CGRP and NO from capsaicin-sensitive afferent nerves. Further, the submucosal application of CGRP induced dose-dependent dilation of gastric submucosal arterioles, which was significantly decreased by L-NAME [23]. However, the dilation induced by submucosal CGRP was decreased to a much lesser degree by inhibition of NO synthesis than that induced with intragastric capsaicin [14]. This indicates that NO released by CGRP is not the only source of submucosal NO in capsaicin-induced vasodilation, and there may be other source of submucosal NO, such as from nitroxidergic nerves that has not been determined thus far [29]. The existence of capsaicin-sensitive nitroxidergic nerves is hypothesized by some reports, but it was not proved [6].

NO is synthesized from L-arginine by NOS in various cells. NOS is present not only in vascular endothelial cells (eNOS) but also in perivascular nerves (nNOS) [10, 30]. It is well known that $\mathrm{NO}$ is a vasodilator that increases gastric mucosal blood flow, and this gas mediator is important in the modulation of gastric mucosal integrity through interaction with sensory nerves [6]. The increase in gastric mucosal blood flow responses to capsaicin is mitigated by 
$N^{\mathrm{G}}$-monomethyl L-arginine, and the gastroprotection induced by capsaicin is also decreased by the NOS inhibitor $[8,31]$. Previous studies have shown that gastric hyperemia caused by acid back-diffusion or exogenous CGRP is mediated partly by NO, but which NOS isoform is involved in those hyperemic responses has not been investigated in any of the studies thus far. In addition, the source of NO involved in the actions of capsaicin was not identified [29]. That is because highly selective inhibitors for NOS isoforms had not been developed at that time. We investigated which NOS isoform mediates the increased gastric mucosal blood flow in response to capsaicin by using newly developed NOS inhibitors.

In this study, NPLA and L-NIO were adopted as selective nNOS and eNOS inhibitors, respectively. It was reported that the potency of inhibition for nNOS by NPLA is 3158 times that for iNOS and 149-fold that for eNOS, whose selectivity for nNOS/iNOS and nNOS/eNOS are ratio of the inverse of $\mathrm{IC}_{50}$ values [32, 33]. L-NIO and $1400 \mathrm{~W}$ were also reported to be as selective inhibitors for eNOS and iNOS, respectively [34, 35].

Intravenous injection of L-NIO alone of L-NAME alone produced a temporary increase in gastric mucosal blood flow, although intravenous injection of NPLA did not affect the gastric mucosal blood flow. Therefore, eNOS-derived NO homeostatically regulates gastric mucosal blood flow under physiological conditions. In the increased gastric mucosal blood flow in responses to capsaicin, NPLA significantly decreased the maximum response of gastric mucosal blood flow during the application of capsaicin, but NPLA did not affect the increase in gastric mucosal blood flow after removal of capsaicin. In contrast, L-NIO significantly decreased gastric mucosal blood flow responses after removal of capsaicin, but did not affect the maximum response of gastric mucosal blood flow induced by capsaicin during the application. These results suggested that $\mathrm{nNOS} / \mathrm{NO}$, which may be released from TRPV1expressing nerves stimulated by capsaicin, plays an important role in gastric hyperemia during the luminal application of capsaicin (as in the early phase). eNOS/NO plays a role in regulating gastric mucosal blood flow responses after removal of capsaicin (as in the late phase). On the other hand, intravenous injection of $1400 \mathrm{~W}$ did not affect the baseline value of gastric mucosal blood flow as well as the increase in gastric mucosal blood flow in response to capsaicin.

Our results were consistent with those reported previously that eNOS was expressed mostly in the vasculature throughout the gastric mucosa in rats, but the expression of iNOS was hardly observed in the gastric mucosa of normal rats by using immunohistochemical analysis [11, 12]. These findings support our hypothesis that eNOS/NO plays a role in the resting state responses of gastric mucosal blood flow in the late phase, while iNOS/NO plays no role in the increased GMBF in response to capsaicin.

In our immunohistochemical studies, TRPV1 immunoreactivity was detected in mucosal and submucosal layers of the corpus of the rat stomach. Many TRPV1 nerve fibers contain nNOS, but not all axons do. In addition, numerous TRPV1-immunoreactive axons were also found around arterioles in the submucosal layer. These data led us to speculate that the capsaicininduced increase in gastric mucosal blood flow could be attributed to the time-dependent interaction of both nNOS/NO and eNOS/NO. Namely, it is suggested that capsaicin stimulates TRPV1 nerves and releases CGRP and NO from the sensory nerve endings. The NO is immediately provided by nNOS and dilates the blood vessels in the early phase. On the other 
hand, NO derived from eNOS, but not nNOS, maintains the persistent dilation of the blood vessels after capsaicin application in rat stomachs (in the late phase) (Fig. 9).

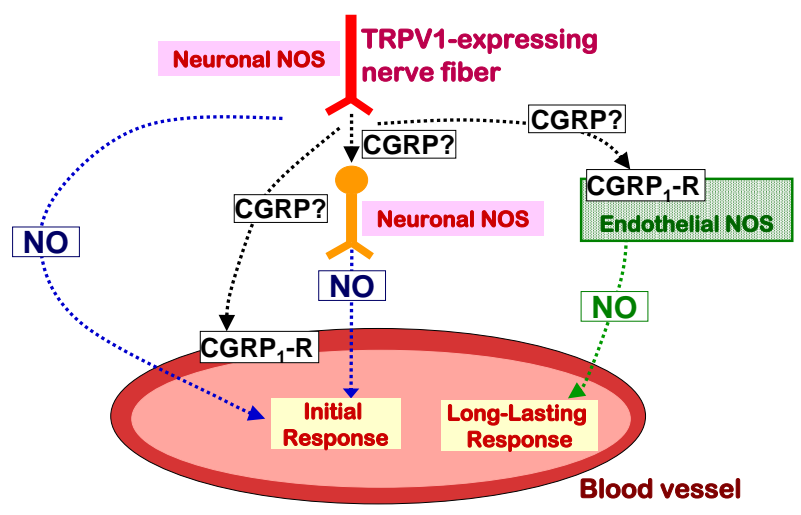

Figure 9. Putative mechanism underlying increase of gastric mucosal blood flow due to activation of TRPV1 channels. Capsaicin increases blood flow in the rat stomach by a peripheral axon reflex-like circuit. Capsaicin excites primary afferent neurons that originate in the dorsal root ganglia and that arborize into several branches that innervate mucosa and submucosal arterioles. The reflex hyperemia is mediated by calcitonin gene-related peptide (CGRP) and nitric oxide (NO) that form most likely in the endothelium. The involvement of NO derived from nNOS in hyperemia is newly clarified. The primary afferent neurons release NO/nNOS to dilate blood vessels.

Stimulation of sensory nerves leads to gastric hyperemic response, which is mediated partly by NO formation, but it is not clear whether the NO is derived solely from endothelial cells or is also released directly by extrinsic and/or intrinsic nerves [6, 14]. We have reported that abundant TRPV1 axons containing nNOS in the submucosa with a high density of immunoreactive axons around blood vessels [21]. Therefore, our study suggests that capsaicin is likely to induce vasodilatation by inducing NO release from TRPV1-expressing sensory fibers.

\section{Conclusion}

The mucosal application of capsaicin increased gastric mucosal blood flow by releasing NO derived from nNOS after stimulating capsaicin-sensitive sensory nerves. The contribution of nNOS-derived NO in gastric hyperemic responses to the activation of TRPV1 by capsaicin has been reported using not only pharmacological but also immunohistochemical techniques. We also demonstrated the interaction between $\mathrm{nNOS} / \mathrm{NO}$ and eNOS/NO on the increased gastric mucosal blood flow by capsaicin in time-dependently, whose hyperemic responses were composed of the both $\mathrm{nNOS} / \mathrm{NO}$ in the early phase and eNOS/NO in the late phase. 


\section{Terminology}

1400W: a selective inducible NOS inhibitor

ANOVA: analysis of variance

BCTC: $N$-(4-t-butylphenyl)-4-(3-chloropyridin-2-yl)tetrahydropyrazine-1(2H)-carboxamide: a TRPV1 antagonist

CGRP: calcitonin gene-related peptide

GMBF: gastric mucosal blood flow

iNOS: inducible NO synthase

eNOS: endothelial NO synthase

L-NIO: $N^{5}$-(l-iminoethyl)-L-ornithine: a selective endothelial NOS inhibitor

nNOS: neuronal NO synthase

NO: nitric oxide

NPLA: $N^{5}$-[imino (propylamino) methyl]-L-ornithine: a selective nNOS inhibitor

TRPV1: transient receptor potential vanilloid type 1

S.E.M.: standard error of mean

\section{Acknowledgements}

This work was supported by Grants-in-Aid for Scientific Research from the Ministry of Education, Culture, Sports and Technology, Japan.

\section{Author details}

Syunji Horie ${ }^{1 *}$, Masaki Raimura ${ }^{2}$, Kenjiro Matsumoto ${ }^{1}$, Takao Namiki $^{2}$, Katsutoshi Terasawa ${ }^{3}$, John V. Priestley ${ }^{4}$ and Kimihito Tashima ${ }^{1}$

*Address all correspondence to: shorie@jiu.ac.jp

1 Laboratory of Pharmacology, Faculty of Pharmaceutical Sciences, Josai International University, Togane, Japan

2 Department of Frontier Japanese-Oriental (Kampo) Medicine, Graduate School of Medicine, Chiba University, Chiba, Japan

3 Department of Kampo Medicine, Chiba Central Medical Center, Chiba, Japan 
4 Neuroscience Centre, St. Bartholomew's and the Royal London School of Medicine and Dentistry, Queen Mary University of London, London, United Kingdom

Kimihito Tashima, Laboratory of Pharmacology, Faculty of Pharmaceutical Sciences, Josai International University, Togane, Japan

The authors have no competing interests.

\section{References}

[1] Lippe IT, Pabst MA, Holzer P: Intragastric capsaicin enhances rat gastric acid elimination and mucosal blood flow by afferent nerve stimulation. Br J Pharmacol 1989; 96: $91-100$

[2] Matsumoto J, Takeuchi K, Okabe S: Characterization of gastric mucosal blood flow response induced by intragastric capsaicin in rats. Jpn J Pharmacol 1991; 57: 205-213

[3] Caterina MJ, Schumacher MA, Tominaga M, Rosen TA, Levine JD, Julius D: The capsaicin receptor: a heat-activated ion channel in the pain pathway. Nature 1997; 389: 816-824

[4] Horie S, Yamamoto H, Michael GJ Uchida M, Belai A, Watanabe K, Priestley JV, Murayama T: Protective role of vanilloid receptor type 1 in $\mathrm{HCl}$-induced gastric mucosal lesions in rats. Scand J Gastroenterol 2004; 39: 303-312

[5] Tashima K, Nakashima M, Kagawa S, Kato S, Takeuchi K: Gastric hyperemic response induced by acid back-diffusion in rat stomachs following barrier disruptionrelation to vanilloid type-1 receptors. Med Sci Monit 2002; 8: BR157-BR163

[6] Holzer P: Neural emergency system in the stomach. Gastroenterology 1998; 114: 823839

[7] Lambrecht N, Burchert M, Respondek M, Müller KM, Peskar BM: Role of calcitonin gene-related peptide and nitric oxide in the gastroprotective effect of capsaicin in the rat. Gastroenterology 1993; 104: 1371-1380

[8] Whittle BJ, Lopez BJ, Moncada S: Nitric oxide mediates rat mucosal vasodilation induced by intragastric capsaicin. Eur J Pharmacol 1992; 218: 339-341

[9] Wallace JL, Miller MJ: Nitric oxide in mucosal defense: a little goes a long way. Gastroenterology 2000; 119: 512-520

[10] Knowles RG, Moncada S: Nitric oxide synthases in mammals. Biochem J 1994 298: 249-258

[11] Price KJ, Hanson PJ, Whittle BJ: Localization of constitutive isoforms of nitric oxide synthase in the gastric glandular mucosa of the rat. Cell Tissue Res 1996; 285: 157-163 
[12] Kato S, Kawahara R, Yasuda M, Amagase K, Takeuchi K: Aggravation of cold-restraint stress-induced gastric lesions in adjuvant arthritic rats: pathogenic role of inducible and endothelial nitric oxide. J Pharmacol Sci 2009; 111: 244-252

[13] Phillipson M, Henriksnäs J, Holstad M, Sandler S, Holm L: Inducible nitric oxide synthase is involved in acid-induced gastric hyperemia in rats and mice. Am J Physiol 2003; 285: G154-G162

[14] Chen RY, Guth PH: Interaction of endogenous nitric oxide and CGRP in sensory neuron-induced gastric vasodilation. Am J Physiol 1995; 268: G791-G796

[15] Takeuchi K, Ishihara Y, Okada M, Niida H, Okabe S: A continuous monitoring of mucosal integrity and secretory activity in rat stomach: a preparation using a lucite chamber. Jpn J Pharmacol 1989; 49: 235-244

[16] Pomonis JD, Harrison JE, Mark L, Bristol DR, Valenzano KJ, Walker K: N-(4-Tertiarybutylphenyl)-4-(3-cholorphyridin-2-yl)tetrahydropyrazine-1(2H)-carbox-amide (BCTC), a novel, orally effective vanilloid receptor 1 antagonist with analgesic properties: II. in vivo characterization in rat models of inflammatory and neuropathic pain. J Pharmacol Exp Ther 2003; 306: 387-393

[17] Esplugues JV, Ramos EG, Gil L, Esplugues J: Influence of capsaicin-sensitive afferent neurons on the acid secretory responses of the stomach in vivo. Br J Pharmacol 1990; 100: 491-496

[18] Yonei Y, Holzer P, Guth PH: Laparotomy-induced gastric protection against ethanol injury is mediated by capsaicin-sensitive sensory neurons. Gastroenterology 1990; 99: 3-9

[19] Horie S, Michael GJ, Priestley JV: Co-localization of TRPV1-expressing nerve fibers with calcitonin-gene-related peptide and substance $\mathrm{P}$ in fundus of rat stomach. Inflammopharmacology 2005;13(1-3):127-37.

[20] Ward SM, Bayguinov J, Won KJ, Grundy D, Berthoud HR: Distribution of the vanilloid receptor (VR1) in the gastrointestinal tract. J Comp Neurol. 2003; 465(1): 121-135

[21] Raimura M, Tashima K, Matsumoto K, Tobe S, Chino A, Namiki T, Terasawa K, Horie S: Neuronal nitric oxide synthase-derived nitric oxide is involved in gastric mucosal hyperemic response to capsaicin in rats. Pharmacology 2013; 92(1-2):60-70.

[22] Saeki T, Ohno T, Boku K, Saigenji K, Katori M, Majima M: Mechanism of prevention by capsaicin of ethanol-induced gastric mucosal injury--a study in the rat using intravital microscopy. Aliment Pharmacol Ther 2000; Suppl 1: 135-144

[23] Chen RY, Li DS, Guth PH: Role of calcitonin gene-related peptide in capsaicin-induced gastric submucosal arteriolar dilation. Am J Physiol 1992; 262: H1350-H1355 
[24] Holzer P, Livingston EH, Guth PH: Sensory neurons signal for an increase in rat gastric mucosal blood flow in the face of pending acid injury. Gastroenterology 1991; 101:416-423.

[25] Vanner S, Bolton M: Neural circuitry of capsaicin-sensitive afferents innervating submucosal arterioles in guinea pig ileum. Am J Physiol 1996;270:G948-G955

[26] Pique JM, Whittle BJ, Esplugues JV: The vasodilator role of endogenous nitric oxide in the rat gastric microcirculation. Eur J Pharmacol 1989; 174:293-296

[27] Tepperman BL, Whittle BJ: Endogenous nitric oxide and sensory neuropeptides interact in the modulation of the rat gastric microcirculation. Br J Pharmacol 1992; 105:171-175

[28] Takeuchi K, Kato S, Takeeda M, Ogawa Y, Nakashima M, Matsumoto M: Facilitation by endogenous prostaglandins of capsaicin-induced gastric protection in rodents through EP2 and IP receptors. J Pharmacol Exp Ther 2003; 304: 1055-1062

[29] Holzer P: Neural regulation of gastrointestinal blood flow. In: Johnson LR (ed.) Physiology of the Gastrointestinal Tract, Fourth Eddition, Academic Press; 2006. p817-839.

[30] Moncada S, Palmer RMJ, Higgs A: Nitric oxide: physiology, pathophysiology, and pharmacology. Pharmacol Rev 1991; 43: 109-142

[31] Whittle BJ, Lopez BJ, Moncada S: Regulation of gastric mucosal integrity by endogenous nitric oxide: interactions with prostaglandins and sensory neuropeptides in the rat. Br J Pharmacol 1990; 99: 607-611

[32] Cooper GR, Mialkowski K, Wolff DJ: Cellular and enzymatic studies of N $\omega$-propylL-ariginine and S-Ethyl-N-[4-trifluoromethyl phenyl]isothiourea as reversible, slowly dissociating inhibitors selective for the neuronal nitric oxide synthase isoforms. Arch Biochem Biophys 2000; 375: 183-194

[33] Zhang HQ, Fast W, Marletta MA, Martasek P, Silverman RB: Potent and selective inhibition of neuronal nitric oxide synthase by $\mathrm{N}$ omega-propyl-L-arginine. J Med Chem 1997; 40:3869-3870

[34] Wolff DJ, Lubeskie A, Gauld DS, Neulander MJ: Inactivation of nitric oxide synthases and cellular nitric oxide formation by $\mathrm{N}^{6}$-iminoethyl-L-lysine and $\mathrm{N}^{5}$-iminoetyl-L-irnithine. Eur J Pharmacol 1998; 350: 325-334

[35] László F, Whittle BJ: Actions of isoform-selective and non-selective nitric oxide synthase inhibitors on endotoxin-induced vascular leakage in rat colon. Eur J Pharmacol 1997; 334:99-102 
